\title{
Editorial
}

\section{Advanced Modeling and Services Based Mathematics for Ubiquitous Computing}

\author{
Jong Hyuk Park, ${ }^{1}$ Hong Shen, ${ }^{2}$ Jian-nong Cao, ${ }^{3}$ Fatos Xhafa, ${ }^{4}$ and Young-Sik Jeong ${ }^{5}$ \\ ${ }^{1}$ Department of Computer Science \& Engineering, Seoul National University of Science \& Technology, Seoul 139-743, Republic of Korea \\ ${ }^{2}$ School of Computer Science, University of Adelaide, Adelaide, SA 5000, Australia \\ ${ }^{3}$ Department of Computing, Hong Kong Polytechnic University, Hung Hom, Kowloon, Hong Kong \\ ${ }^{4}$ Department of Computer Science, Technical University of Catalonia, 08034 Barcelona, Spain \\ ${ }^{5}$ Department of Multimedia Engineering, Dongguk University, Seoul 100-715, Republic of Korea
}

Correspondence should be addressed to Young-Sik Jeong; ysjeong.dk@gmail.com

Received 8 June 2015; Accepted 8 June 2015

Copyright (C) 2015 Jong Hyuk Park et al. This is an open access article distributed under the Creative Commons Attribution License, which permits unrestricted use, distribution, and reproduction in any medium, provided the original work is properly cited.

\section{Introduction}

Recent advancements on Ubiquitous Computing have been a great challenge to computer science and engineering. Ubiquitous Computing systems manage huge numbers of heterogeneous mobile devices which continuously connect real-world objects, and most data are automatically generated through wireless communication environments [1-3]. Ubiquitous Computing frameworks might help support the interaction between "humans and objects" and allow for more complex structures like intelligent computing and application development. Many Ubiquitous Computing frameworks seem to focus on real time data logging solutions which offer some basis to work with many "humans and objects." Future developments might lead to specific software development environments to create the software and to work with the hardware used in the Ubiquitous Computing [1-5].

This special issue aims to provide an advanced theory and application for helping researchers to conduct new research and reviewing articles that present latest and practical findings that can contribute to the future evolutions of mathematics in Ubiquitous Computing applications.

Among many manuscripts we have received, only high quality manuscripts were finally selected for this special issue. Each selected manuscript was blindly reviewed by at least three reviewers consisting of guest editors and external reviewers. We present a brief overview of each manuscript in the following.

\section{Related Works}

Recent advancements in advanced modeling and services based mathematics for Ubiquitous Computing have created new research topics including (1) Mathematical and numerical modeling for Ubiquitous Computing, (2) optimization methods, mathematics modeling, and services for Ubiquitous Computing, (3) numerical analysis for security and emergencies for Ubiquitous Computing, (4) methods for improving efficiency or accuracy of M2M applications, (5) vehicle autodiagnosis for Ubiquitous Computing, (6) computational models of communication mechanisms for Ubiquitous Computing, (7) adaptive and dynamic algorithms for Ubiquitous Computing, (8) applied cryptography and security issues for Ubiquitous Computing, (9) advanced modeling and services for IoT (Internet of Things) applications, and (10) ubiquitous sensor networks and RFID for Ubiquitous Computing.

In these several topics of this special issue, some articles proposed the following findings.

$\mathrm{N}$. Javaid et al. proposed the routing model overhead produced by three reactive protocols; AODV, DSR, and DYMO. They choose the three routing protocols because these are widely used in literature. Their main focus was to measure 
routing overhead for LL and NL feedback mechanisms. To analyze the link sensing mechanisms of AODV, DSR, and DYMO, they conducted simulations in NS-2. The overhead was measured for nodes' different mobility and densities.

M. Aazam and E.-N. Huh proposed a resource management model, keeping in view different types of services, different customer types, customer characteristic, pricing, and refunding. The presented model was implemented and evaluated using CloudSim 3.0.3 toolkit. Their results and discussion validated the model and its efficiency.

S. Lee et al. proposed a NUI-specific SDK, called "Gesture SDK," for the development of NUI-based applications. Gesture SDK provides a gesture generator with which developers can directly define gestures. Further, a "Gesture Recognition Component" was provided, which enables defined gestures to be recognized by applications. They generated gestures using the proposed SDK and developed a "Smart Interior," NUI-based application using the Gesture Recognition Component. The results indicated that the recognition rate of the generated gestures was $96 \%$ on average.

S. Liu and X. Meng constructed a Region-Based Location Graph (RLG), which can combine with user shortranged mobility formed by daily activity and long-distance mobility formed by social network tie. They can sequentially recommend local business information and long-distance business information to users. Moreover, it can combine userbased collaborative filtering with item-based collaborative filtering and successfully generate recommendation for cold start users. Consequently it can alleviate cold start problem which traditional recommender systems often suffer from. The experiments on real dataset confirmed the effectiveness of the proposed method compared to other ones.

H. Kim et al. implemented a mobile diagnosing system that provides user-centered interfaces for more precisely estimating and diagnosing engine conditions through communications with the self-developed ECU only for industrial CRDI engine use. For the implemented system, a new protocol was designed and applied based on OBD-II standard to receive engine data values of the developed ECU.

I. Y. Jung et al. proposed a security framework for health information management of the self-organizing software platform (SoSp). The proposed framework was designed to ensure easy detection of identification information for typical users. In addition, it provides powerful protection for the user's health information.

H. Kim et al. proposed a new framework for generating 3D city models that satisfy both the visual and physical requirements for ground-oriented virtual reality applications. To ensure its usability, the framework must be cost-effective and allow for automated creation. To achieve these goals, they leveraged a mobile mapping system that automatically collects high-resolution images and supplements sensor information such as the position and direction of the captured images. To resolve problems stemming from sensor noise and occlusions, they developed a fusion technique to incorporate digital map data. This paper described the major processes of the overall framework and the proposed techniques for each step and presented experimental results from a comparison with an existing 3D city model.

G. Yi and N. Y. Yen designed a tool for visualizing social network data from the famous social networking website Facebook. It also proposed a new interaction and navigation technique that uses Kinect to explore and interact with social networks [6]. The tool as well as the new method of interaction should help users in interacting and exploring their social networks. They would also help learners experience better learning interaction.

A. Mahmud et al. proposed a medium access control protocol that can work in the absence of a CCC and reduce the possible overhead to a greater extent. In their proposed protocol, CR users took advantage of similar spectrum availability in their neighborhood for resource utilization. They also proposed a contention-based spectrum allocation mechanism that works in a distributed manner over different available channels. Simulation results showed that this approach can reduce broadcast overhead significantly while maintaining connectivity success similar to its counterparts.

J. Sim et al. examined the influence of dataset characteristics and patterns of missing data on the performance of classification algorithms using various datasets. The moderating effects of different imputation methods, classification algorithms, and data characteristics on performance were also analyzed. The results were important because they could suggest which imputation method or classification algorithm to use depending on the data conditions. The goal was to improve the performance, accuracy, and time required for Ubiquitous Computing.

K.-C. Lin et al. proposed a feature selection model combining the modified AFSA (MAFSA) with SVM. MAFSA was used to simulate the mechanism underlying the endocrine system in order to create a different search space for every individual fish in order to enhance the efficiency with which optimal solutions are derived.

G. Yi conducted clustering under the assumption that the functional classifier inside the cluster had similar functions and utilized the features extracted from the inside of the cluster as the learning data. When finding protein whose function is unknown, the model that predicts GO (or the controlled vocabulary) was defined through the learning and learned data documents of those proteins whose function was already defined. This was the existing functional prediction, which is the method to harmonize appropriately those frequently used methods such as sequence similarity, proteininteraction, and context-free ones; thus, it could increase the prediction probability of GO.

J.-R. Chang and B. Chang developed a model to investigate the tourists' preference. Ten attributes of tourist destinations were used in this study. Fuzzy set theory was adopted as the main analysis method to find the tourists' preference. In their research, 248 types of data were used. Besides the evaluations for the factors, the overall evaluations (namely, satisfied, neutral, and dissatisfied) for every tourism destination were also inquired. After screening, 201 types of these data were usable. Among these 201 types of data, $141(70.15 \%)$ were classified into "satisfied" with the tourism destination, 49 (24.38\%) were "neutral," and 11 (5.47\%) were 
"dissatisfied." Eight rules were obtained with the method of fuzzy preprocess. Regarding the condition attributes, three of the original 10 attributes were found influential, namely, level of prices, living costs, information, and tourist services as well as tourist safety of the tourism destinations. These study results showed that top management of tourism destinations should put resources in these fields first, in order to allow limited resources to perform to their maximum effectiveness.

Y. Zhang et al. proposed a new efficient and provable secure digital rights management authentication scheme using smart card based on elliptic curve cryptography. To demonstrate the scheme is provable secure, they introduced a security model AFP05 and analyzed the scheme in this model. In the following, they gave the proof that the proposed scheme was secure in the AFP05 model. As known to all, one-way hash function is more efficient than the operation of scalar multiplication and pairings. Moreover, the pairing operation costs much more than the scalar multiplication operation. The effort of evaluating one pairing operation is approximately three times the effort of evaluating one scalar multiplication operation. So, they cut down some pairings operation of point on elliptic curve and used hash function instead to increase the scheme's efficiency.

J. Uddin et al. proposed a method for the reliable fault detection and classification of induction motors using twodimensional (2D) texture features and a multiclass support vector machine (MCSVM). The proposed model first converts time-domain vibration signals to $2 \mathrm{D}$ gray images and then utilizes the global neighborhood structure (GNS) map to extract texture features of the converted gray images. GNS maps were calculated by averaging the local neighborhood structure (LNS) maps of central pixels. The principle component analysis (PCA) is then used to select the most significant feature dimensions.

H. Cho and B. Kim suggested Improved Cooperative Collision Warning System (ICCWS) that considers the curvature of the road and is based on intervehicle communication. To predict the radius of curvature of the road, the Arc Relative Distance (ARD), the real relative distance to a preceding vehicle on a curved road has been used. The risk of collision with the preceding vehicle was decided by calculating an index of the risk of collision on a curved road using the computed ARD. The effect of ICCWS proposed through this simulation has been reviewed, and the improvement in performance in following a preceding vehicle has been analyzed quantitatively via comparative analysis with the conventional forward collision warning system.

W.-S. Lee et al. proposed a subsurface scattering-based object rendering technique that was optimized for smartphone games. They employed a subsurface scattering method that is utilized for a real time smartphone game. Their example game was designed to validate how the proposed method can be operated seamlessly in real time. Finally, they showed the comparison results between bidirectional reflectance distribution function, bidirectional scattering distribution function, and their proposed subsurface scattering method on a smartphone game.

Finally S. Nam and I. Kim presented an efficient spatial reasoning algorithm working on a mixture of directional and topological relations between spatial entities and then explained the implementation of a spatial reasoner based on the proposed algorithm. Their algorithm not only has the checking function for path-consistency within each directional or topological relation set, but also provides the checking function for cross-consistency between them. This paper also presented an application system developed to demonstrate the applicability of the spatial reasoner and then introduced the results of the experiment carried out to evaluate the performance of the spatial reasoner.

\section{Acknowledgment}

We would like to thank all authors for their contributions to this special issue. We also extend our thanks to the external reviewers for their excellent help in reviewing the manuscripts.

$$
\begin{array}{r}
\text { Jong Hyuk Park } \\
\text { Hong Shen } \\
\text { Jian-nong Cao } \\
\text { Fatos Xhafa } \\
\text { Young-Sik Jeong }
\end{array}
$$

\section{References}

[1] M. Friedewald and O. Raabe, "Ubiquitous computing: an overview of technology impacts," Telematics and Informatics, vol. 28, no. 2, pp. 55-65, 2011.

[2] Y.-S. Jeong, N. Chilamkurti, and L. J. García Villalba, "Advanced technologies and communication solutions for internet of things," International Journal of Distributed Sensor Networks, vol. 2014, Article ID 896760, 3 pages, 2014.

[3] Gartner, Gartner's Hype Cycle Special Report for 2011, Gartner, 2012, http://www.gartner.com/technology/research/hypecycles/.

[4] H. Ning and Z. Wang, "Future internet of things architecture: like mankind neural system or social organization framework?" IEEE Communications Letters, vol. 15, no. 4, pp. 461-463, 2011.

[5] Y.-S. Jeong and J. H. Park, "High availability and efficient energy consumption for cloud computing service with grid infrastructure," Computers and Electrical Engineering, vol. 39, no. 1, pp. 15-23, 2013.

[6] R. Francese, I. Passero, and G. Tortora, "Wiimote and Kinect: gestural user interfaces add a natural third dimension to HCI," in Proceedings of the International Working Conference on Advanced Visual Interfaces (AVI '12), pp. 116-123, May 2012. 


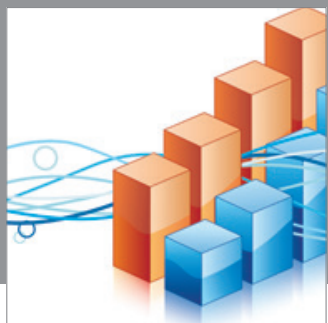

Advances in

Operations Research

mansans

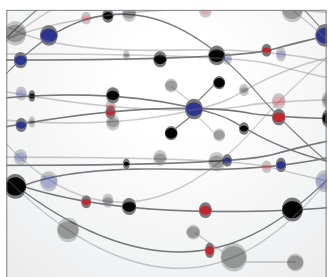

The Scientific World Journal
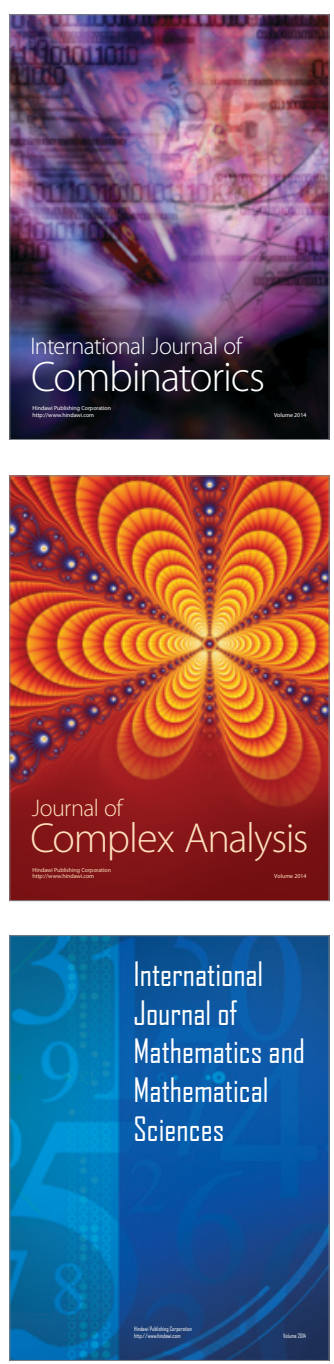
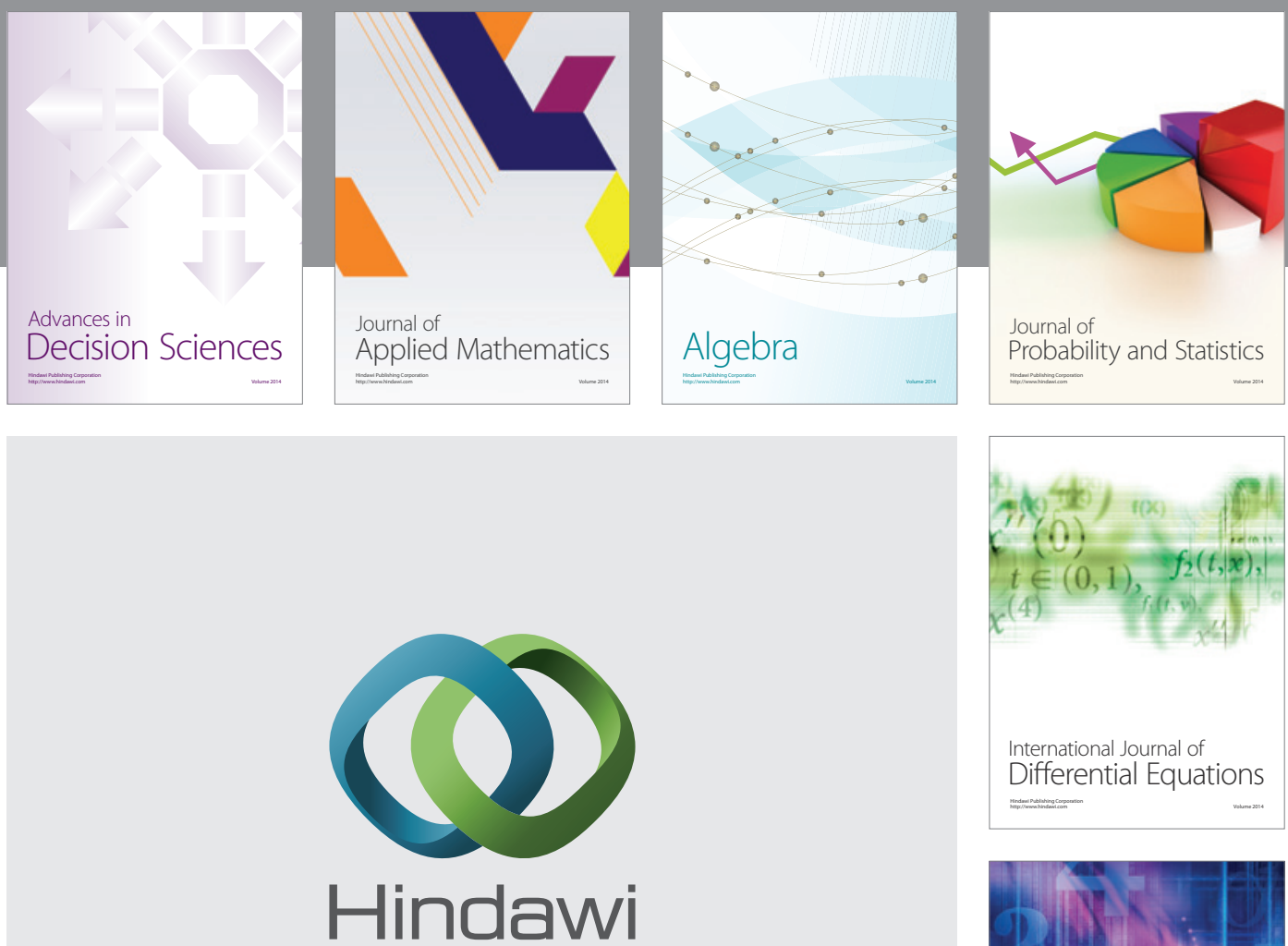

Submit your manuscripts at http://www.hindawi.com
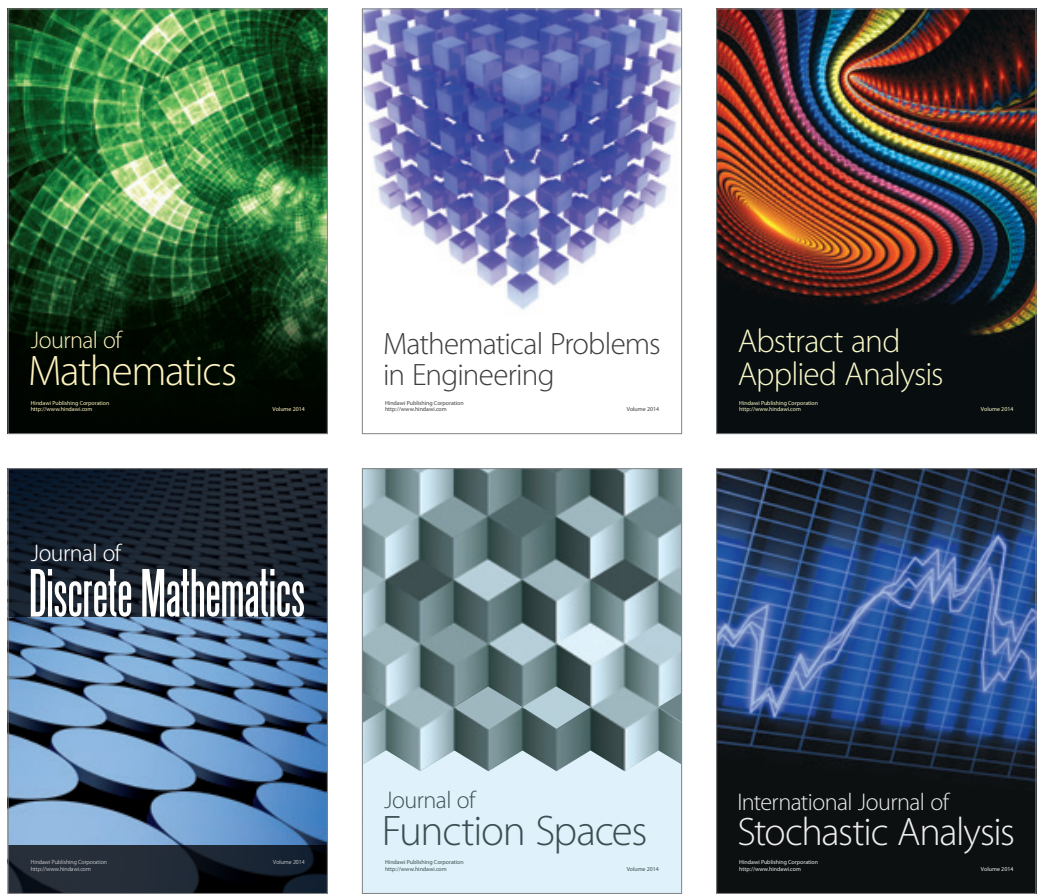

Journal of

Function Spaces

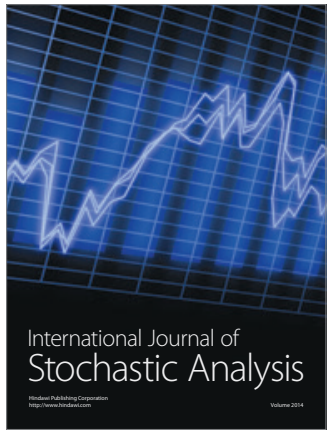

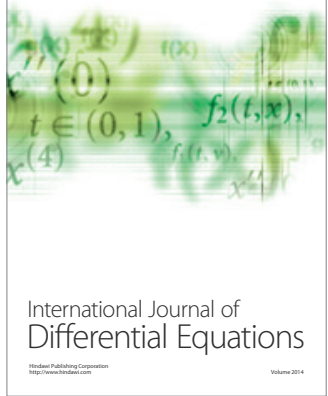
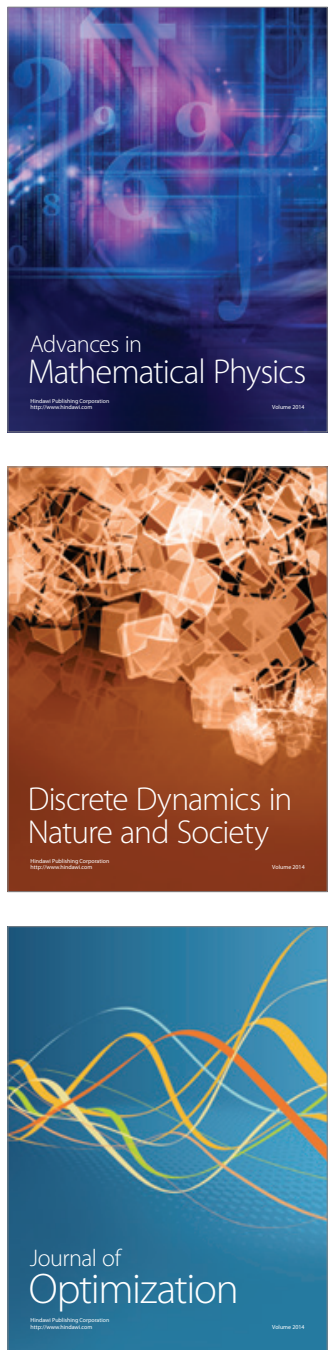